\title{
Editorial
}

\section{Mathematical models of liquid crystals}

A symposium held in Durham in July 1995 brought together an international collection of key mathematicians, theoretical physicists and experimentalists in the areas of liquid crystals and polymeric systems. Many of the participants met together for the first time, and the symposium stimulated new collaborative interactions among applied mathematicians and others who, prior to this meeting, were working separately on either liquid crystals or polymer fluids. The symposium also enhanced further interchanges of ideas between industry and academia. The flavour of this meeting is captured by the contents of this special issue which resulted from presentations given by invited speakers.

The first four papers are principally concerned with the mathematical modelling of nematic and smectic liquid crystals. Liquid crystals are anisotropic media which usually consist of elongated molecules whose average preferred common direction in space is often described by the unit vector $\boldsymbol{n}$, called the director or orientation field. Smectic $C$ liquid crystals are made up of layers where the director $\boldsymbol{n}$ makes an angle $\theta$ with respect to the layer normal. The orientation of $\boldsymbol{n}$ within a sample is affected by the application of electric or magnetic fields (such a reorientation is called a Freedericksz transition), and is described by differential equations arising from the minimization of an energy function $W$ which depends on $\boldsymbol{n}$ and its gradients. Knowing the solution to these equations allows a complete description of the orientation alignment of a sample of liquid crystal which, in turn, allows, for example, the direction of light through a device to be controlled.

The paper by Atkin and Stewart examines theoretical predictions for Freedericksz transitions for smectic $\mathrm{C}$ liquid crystals in some basic sample geometries using recently derived continuum theory. Blake, Leslie and Towler discuss switching and induced flow for chiral smectic C liquid crystals in planar aligned samples in the well known 'bookshelf' geometry. In both these papers, the smectic layers are assumed to be equidistant. An extension of the continuum theory to allow layer dilation or compression is presented in the article by McKay and Leslie. The paper by Brown, Dunn and Jones presents experimental measurements for two of the smectic elastic constants which appear in the continuum theory contained, for example, in the first two papers of this special issue. These results are important for optimizing the liquid crystal materials used in electro-optic devices and demonstrate the potential for future modelling.

The final two papers deal with different aspects of nematic liquid crystals. In place of the usual Freedericksz transitions induced by electric or magnetic fields, these papers describe mathematically the orientations of the director $\boldsymbol{n}$ which arise from statics or flow properties; this is in contrast to the approach mainly discussed in the first papers of this issue. The static equilibrium configuration of the interface between a nematic liquid crystal 
and an isotropic medium is discussed via surface energies by Faetti and Virga. Their formulation also allows the construction of an equilibrium equation for the static configuration along edges for a nematic at such a fixed interface. The final paper by Calderer examines the non-Newtonian flow properties of polymeric nematic liquid crystals via the theory of Ericksen, which incorporates an order parameter $s$ in addition to the usual director $\boldsymbol{n}$; this approach is a natural extension of previous nematic theories and various configurations involving Poiseuille flow are discussed.

The Durham symposium owes much to the guidance of the London Mathematical Society and, at Durham University, Professor A. J. Scholl, Dr J. Bolton and Mrs S. Nesbitt. The support received involving local arrangements, correspondence with participants, etc., was of great assistance to the symposium organizers. The organizers also wish to express their thanks to the EPSRC for financial support which enabled this symposium to take place, and to Cambridge University Press for publishing this special issue. Finally we wish to express our thanks to our fellow organizers, Professor T. C. B. McLeish and K. Walters, FRS.

\section{F. M. Leslie, FRS \\ I. W. Stewart Strathclyde}

